\title{
Advancements in Protein based Nano Particulate system for treatment of Pulmonary Infections- A Review
}

\author{
Praveen Tahilani*, D P Chatterjee
}

Department of Pharmaceutical Science, SAGE University, Indore Madhya Pradesh, India

\author{
*Correspondence \\ Mr. Praveen Tahilani, \\ M-Pharm. (Pharmaceutics), PhD* \\ Department of Pharmaceutical Science, SAGE University, Indore Madhya Pradesh, India. \\ E-Mail: tahilanipraveen@gmail.com
}

\begin{abstract}
In addition to the so-called small molecule drugs, proteins and peptides are of increasing interest for pharmacotherapy, due to several advantageous properties. In general, those compounds are administered parenterally. However, non-invasive routes of administration represent a great part of research. Amongst others is the pulmonary application of proteins and peptides for local delivery in the case of pulmonary diseases, such as idiopathic pulmonary fibrosis, where the alveolar epithelium is affected. To ensure an intracellular delivery, nano particles in a size range of $150 \mathrm{~nm}$ will be prepared via charge-mediated coacervation, characterized for their physicochemical properties and loaded with several model-proteins. The material used for nano particle preparation was chosen to be positively and negatively charged starch derivatives, which were synthesized from potato starch. Although nano particles in that size range are known to show an increased cell uptake, they do not show a high deposition in the deep lung. Thus, an advanced carrier system consisting of a fast dissolving micro particle matrix with embedded starch nano particles will be developed and characterized. Due to its aerodynamic properties, that carrier system must be able to deposit a high fraction of the applied dose in the deep lung $(\sim 50 \%)$, while at the same time demonstrating (in in vitro models) the ability to facilitate uptake of starch nano particles into cells of the alveolar epithelium after fast dissolution of the micro particle matrix.
\end{abstract}

Keywords: Pulmonary diseases, charge-mediated coacervation, advanced carrier system, nano particles, aerodynamic properties.

This is an Open Access article that uses a fund-ing model which does not charge readers or their institutions for access and distributed under the terms of the Creative Commons Attribution License (http://creativecommons.org/licenses/by/4.0) and the Budapest Open Access Initiative (http://www.budapestopenaccessinitiative.org/read), which permit unrestricted use, distribution, and reproduction in any medium, provided the original work is properly credited.

\section{Introduction}

In recent years, there has been a drastic increase in research, technological development at atomic, and molecular and macromolecular scales which lead to the controlled manipulation and study of structure ranges from 1 to $100 \mathrm{~nm}$. Nano particles fit in to the category of colloidal drug delivery system which behaves like a whole unit with respect to its properties and transport mechanism. These are used as drug carrier system to improve the cellular uptake as well as body distribution. Nano particles became the major reason for the change in different properties of many conventional materials by virtue of their greater surface area per weight than micro particles which makes them to be more active drug carriers. Several types of nano particulate system which includes polymeric nano particles, polymeric micelles, solid nano particles, lipid based nano particles, for example, solid lipid nano particles(SLN), nano structured lipid carriers (NLC), and lipid drug conjugate (LDC), liposomes, inorganic nano particles, dendrimers, magnetic nano particles, nano crystals, and nano tubes. Various types of materials are used to prepare the nano particles which include polymers, lipids, polysaccharides and proteins.There are some criteria for selection of matrix material for nano particles which are size of the nano

Tahilani \& Chatterjee ASIAN PACIFIC JOURNAL OF NURSING AND HEALTH SCIENCES, 2021; 4(1):-22-34 
particle, desired drug release profile, properties of the drug such as solubility and stability, and nature of the material, that is, biodegradability and toxicity. Recently, biopolymer based nano particles including protein nano particles are actively used in pharmaceuticals and nutraceuticals due to their low toxicity and biodegradability. [1,2]

Historically speaking, active pharmaceutical ingredients (APIs) such as acetylsalicylic acid or paracetamol have been commonly characterized by a small molecular weight. However, modern drug discovery approaches most often lead to drugs with a hydrophobic character and a high molecular weight, resulting in poor water solubility and low permeability over epithelial barriers. In recent times, the use of socalled biopharmaceuticals, including nucleic acid- and protein- based APIs with complex chemical structures have begun to find favor in therapeutic settings; such entities have shown faster and higher success rates in phase two and three clinical trials compared to conventional APIs. This is especially important for pharmaceutical industry with clinical trials being the most expensive part of development.

Although the concept of nanotechnology was defined by Taniguchi in the 1970s, a universally agreed upon interpretation of this term is still unavailable. Typically, a material can be considered as 'nano' if it exists in a size range of $1 \mathrm{~nm}$ to $100 \mathrm{~nm}$, with special physical and chemical properties or biological effects that differ from its larger-scale counterpart. In the field of pharmacy the definition is even broader, including particles up to $1000 \mathrm{~nm}$ in size.

However, there is also a public fear of nanotechnology, with toxicity arising from materials in the nano-size range being an important concern to be addressed. Worst case scenarios from e.g. studies on combustion particles and their effect on the environment or human health are indeed important for risk assessment but should never be extrapolated to nano particles with a deliberate use in humans as for the field of nano medicine. Here, nano particulate system is applied for medical purposes in order to produce the safest possible product, manufactured from high quality raw materials by the best manufacturing processes, and regulated by a quality control of the highest

Standards. The European Technology Platform on Nano Medicine has defined nano medicine as: "The application of nanotechnology in health care, exploiting the improved and often novel physical, chemical, and biological properties of materials at the nano metric scale" This Figure demonstrates the specific position of nano medicine within the broad field of nano particles and illustrates the diversity of such nano particles.

Unlike proteins, peptides mostly only possess a primary structure. Due to disulfide bond formation, they sometimes express a secondary structure, as known from vasopressin (anti diuretic hormone) or insulin.

Proteins fulfill various functions in the human body, ranging from stabilizing tissues and organs (e.g. collagens), to transport, storage of molecules, mediation of receptors, and catalysis of metabolic reactions (enzymes). As a result, they often exhibit post-translational modifications, such as phosphorylation, acetylation or glycosylation, which activates the protein and allow it to carry out its specific function. In contrast, most peptides work as signaling agents and are classified as hormones.

In many cases, diseases are the result of missing or malfunctioning proteins. The idea of protein- or peptide-based APIs is often therefore to replace these compounds. Currently, proteins and peptides represent a large fraction of compounds in drug development pipelines, due to their predictable activity profiles and their highly selective mode of action. Additionally, several formulations incorporating protein or peptide APIs are already on the market, as illustrated in the following.

Insulin is the oldest example of a peptide-based API, used for the treatment of diabetes mellitus type 1 - a metabolic disorder resulting from an absolute insulin deficiency, caused by either defects in insulin action, secretion, or both. Before the discovery of insulin by Banting and Best in the 1920s, this disease in its most severe form often led to death. Today, insulin is usually administered via subcutaneous injection. Different modifications of the molecule, resulting in long-acting and short-acting insulin derivatives, allow for mimicry of the physiological secretion of insulin by the pancreas.

\section{Technical Information}

Colonic delivery refers to targeted delivery of drugs into the lower gastrointestinal tract, which occurs primarily in the large intestine (i.e. colon). The colon is a site where both local and systemic delivery of drugs can take place. Local delivery allows topical treatment of inflammatory bowel disease and systemic drug delivery are mainly used for to treat the hypertension. However, treatment can be made effective if the drugs can be targeted directly into the colon, thereby reducing the systemic side effects.

Targeted drug delivery into the colon is highly desirable for hypertension. The colon specific drug 
delivery system protects the drug i.e. drug release and absorption doesn't occur in the stomach and in the small intestine. The colon is believed to be a suitable absorption site for peptides and protein drugs due to less diversity and intensity of digestive enzymes and comparative proteolytic activity of colon mucosa less than that observed in the small intestine.

Colon specific delivery protects peptide drugs from hydrolysis, and enzymatic degradation in duodenum and jejunum, and eventually releases the drug into ileum or colon which leads to greater systemic bioavailability. The colon having long residence time up to 5days is highly responsive to absorption enhancers.

Site-specific delivery to colon is advantageous in developing many pharmaceutical products and also useful

for the absorption of drugs which are poorly soluble. Colon is mostly advantageous as it has slow transit time, less fluid volume, less digestive enzymes with less motility.

Colon specific delivery ensures direct treatment at disease site with lower dosing and less systemic side effects. Entry of drugs into systemic circulation can also occur via the colon. Not only drug solubility but also the stability of drug in colonic environment is a further factor that warrants attention. The drug could bind in a nonspecific manner to dietary residues, intestinal secretions, mucus or general fecal matter, where the concentration of drug may get reduced. Moreover, the resident micro-flora could also affect colonic performance via degradation of the drug. Colonic delivery refers to targeted delivery of drugs into the lower gastrointestinal tract, which occurs primarily in the large intestine (i.e. colon). The colon is a site where both local and systemic delivery of drugs can take place. Local delivery allows topical treatment of inflammatory bowel disease and systemic drug delivery are mainly used for to treat the hypertension. However, treatment can be made effective if the drugs can be targeted directly into the colon, thereby reducing the systemic side effects.

Targeted drug delivery into the colon is highly desirable for hypertension. The colon specific drug delivery system protects the drug i.e. drug release and absorption doesn't occur in the stomach and in the small intestine. The colon is believed to be a suitable absorption site for peptides and protein drugs due to less diversity and intensity of digestive enzymes and comparative proteolytic activity of colon mucosa less than that observed in the small intestine.
Colon specific delivery protects peptide drugs from hydrolysis, and enzymatic degradation in duodenumand jejunum, and eventually releases the drug into ileum or colon which leads to greater systemic bioavailability. The colon having long residence time up to 5days is highly responsive to absorption enhancers.

Site-specific delivery to colon is advantageous in developing many pharmaceutical products and also useful for the absorption of drugs which are poorly soluble. Colon is mostly advantageous as it has slow transit time, less fluid volume, less digestive enzymes withless motility.

Colon specific delivery ensures direct treatment at disease site with lower dosing and less systemic side effects. Entry of drugs into systemic circulation canalso occur via the colon. Not only drug solubility butalso the stability of drug in colonic environment is a further factor that warrants attention. The drugcould bind in a nonspecific manner to dietary residues, intestinal secretions, mucus or general fecal matter, where the concentration of drug may get reduced. Moreover, the resident micro-flora could also affect colonic performance via degradation of the drug.

Lungs offer a large surface area for absorption, rich blood circulation, better permeability, and limited proteolytic activity, which makes it an ideal route for non-invasive administration of therapeutics. Localized delivery shows great promise not only in the treatment of respiratory diseases, such as asthma, tuberculosis, influenza, cystic fibrosis, chronic obstructive pulmonary disease (COPD), etc., but also reduces the systemic toxicity. Alternatively, systemic drug delivery can be achieved by targeting the alveolar region where the drug can be absorbed through a thin layer of epithelial cells and into the systemic circulation. This leads to enhanced permeability, a rapid onset of action, and avoidance of fi rst-pass metabolism. Moreover, recent advances shows immense potential for effi cient pulmonary delivery of proteins that cannot be taken orally and require parenteral delivery[3].In the process of breathing, the lungs are continuously exposed to materials of various sources and sizes, such as pollen $(20-90 \mu \mathrm{m})$, bacteria $(0.2-200 \mu \mathrm{m})$, and tobacco smoke $(0.01-1 \mu \mathrm{m})$. These airborne particles deposit along the respiratory tract from the conducting upper airways (with the oropharynx, trachea, main bronchi, and terminal bronchioles) down to the respiratory region of the lower airways (with respiratory bronchioles and alveolar sacs). In upper airways, 
particles get rapidly cleared by cilia, where they are swallowed and metabolized.

In general, there are no mathematical equations that establish a relationship between particle velocity and its deposition behavior. Thus, determination of particle trajectories, inertia, and diffusion under the synchronized conditions are essential to envisage the distribution pattern of inhaled particles[4].

\section{Inertial Impaction}

Inertial impaction offers a potential mechanism of deposition for the particles between $2 \mu \mathrm{m}$ to $5 \mu \mathrm{m}$. Inertial impaction delivers the drug in the complex geometry of the upper respiratory tract and conducting airway bifurcations. Stoke's number explains the probability of the particle to diverge from streamline of carrier gas.

\section{Gravitational Sedimentation}

Settlement of the particle under the action of the gravity covers the gravitational sedimentation mechanism for the deposition of the particle to the lungs. Particles sized between $0.5 \mu \mathrm{m}$ to $5 \mu \mathrm{m}$ have been settled by this mechanism. An effective deposition by this mechanism occurs in the small airways and the alveoli but the deposition also occurs in the upper respiratory tract.

\section{Brownian Diffusion}

Deposition by Brownian diffusion occurs by the collisions of the particle with gas molecules. It is a potential mechanism of the deposition for the particles having less than $0.5 \mu \mathrm{m}$ diameter and in the acinar region of the lungs. The particles below $0.01 \mu \mathrm{m}$ are effective in nose, mouth, and pharyngeal airways.

\section{Electrostatic Precipitation}

Electrostatic precipitation results when charged particles approaches oppositely charge surfaces onto airways.

\section{Interception}

Interception acts as a potential mechanism of the particle deposition in small airways and alveoli. Interception involves the deposition of an elongated particle in the gas space while one of its ends touches the alveolar wall under the influence of gravity[5].

\section{Clearance of the Particles}

The upper airways (from the trachea till the tertiary bronchi) are lined with a thick mucus film, which acts as a protective layer in order to trap and clear the particles. The mucociliary movements clear the foreign particles immediately before they can move to lower areas of the lung by either coughing or swallowing[6].The clearance in this region also depends on the number of cilia and the ciliary beat frequency, as well as the quality and quantity of mucus . In the deeper areas of the lungs, i.e., the alveolar region, the transport mechanism is believed to be more complex. The alveolar lining consists of a variety of proteins and lipids, which act as a barrier for the transport of the molecules[7]. Along with the alveolar lining, the tight junctions present at the epithelial cell serve as the primary barrier for the transport to occur. The transporter proteins play a vital role in the transport of the API via active absorption or passive diffusion, depending on the nature and chemical structure of the API. Another important aspect in this region is the clearance of molecules by the alveolar macrophages, which needs to be taken into consideration in the drug transport mechanisms. The molecules that are able to cross the barrier are either taken up by the cells and further absorbed into the systemic circulation or phagocytized by the alveolar macrophages [8,9]

\section{Factors Affecting Drug Bioavailability via} Pulmonary Route.

Fate and bioavailability of administered drug via pulmonary depend on a multitude of factors. Various physiochemical, biological, and formulation parameters have played an important role in the absorption of drugs through the lungs[10].

Diffusion and absorption of drugs from the lungs interface depends on the molecule's lipid solubility, size, degree of ionization, and the area of absorptive surface. It is likely that lipid-soluble drugs will diffuse more rapidly due to the lipoidal nature of the cell membrane. Small molecules tend to penetrate membranes more rapidly than larger ones. Most drugs are weak organic acids or bases, existing in un-ionized and ionized forms in an aqueous environment. The unionized form is usually lipid soluble (lipophilic) and diffuses readily across cell membranes[11,12]

The ionized form has low lipid solubility (but high water solubility - i.e., hydrophilic) and high electrical resistance and thus cannot penetrate cell membranes easily. The proportion of the un-ionized form present (and thus the drug's ability to cross a membrane) is determined by the $\mathrm{pH}$ and the drug's partition coefficient[13]

Alveolar mucosal membrane is highly vascular, permitting the drug to enter the systemic circulation rapidly and to reach its target organ with minimal delay. Drugs may also be administered directly into the target organ, resulting in site-specific therapeutic action of drugs. This is important for the delivery of bronchodilators, anti-inflammatory agents, mucolytics, antiviral agents, anticancer agents, and phospho lipidprotein mixtures in critical and chronic conditions like asthma, bronchiectasis, tuberculosis, cystic fibrosis, and others[14].

Different Approches Used For Pulmonary Drug Delivery

Tahilani \& Chatterjee $\quad$ ASIAN PACIFIC JOURNAL OF NURSING AND HEALTH SCIENCES, 2021; 4(1):22-34 
Appropriate devices are used to deliver the drug to the lungs effectively. Selection of devices depends upon physicochemical and dosing demands associated with the drug substances to be inhaled. A variety of technology is under development to overcome problems associated with conventional aerosol device intended for dry powder inhalation system and liquid inhalation system.

\section{Jet Nebulizers}

Jet nebulizers are the most common type of inhalation device. These are the subset of the more general twin $\mathrm{fl}$ uid atomizers. The operating procedure includes a pressurized air source that supplies high-pressure air, which fl ows through a nozzle where the air acceleration takes place. The pressure near the nozzle is designed so that high speed air flows over a short section of the liquid surface supplied by the liquid feed tube. This is the site where the production of liquid droplet takes place.

The primary determinants of both efficiency and output rate are the ability of the nebulizer to return droplets to nebulizer reservoir for re nebulization. A major determinant of the output rate in a jet nebulizer is the rate at which primary droplets are created, which increases with increase in the flow rate of liquid into droplet production site with all other variables unchanged.

\section{Dry Powder Inhalers}

The purpose of DPI is to insert a prescribed dose of powder aerosol into air inhaled by the patient during a single breath while the powder is prevented from being exposed to ambient air until the patient is ready to inhale. Coughing might be induced by inhalation of large amounts of powder; total amounts of inhaled powder are usually less than 10-20 mg. The powder to be inhaled from DPI must be swept up by the air being inhaled, a process known as "Fuidization." The mechanics of dry powder inhalers are complex for irregularly shaped and rough particles.

Most DPI formulations consist of micronized drug blended with large carrier particles which enhance flow, reduce aggregation, and aid in dispersion. A combination of intrinsic physicochemical properties, size, shape, area, and morphology affects forces of interaction and aerodynamic properties which determine fluidization, dispersion, and delivery to lungs and deposition in peripheral airways. On actuation, the formulation gets fluidized and enters the airway. The drug separates from the carrier and gets carried deep into the lungs. The larger carrier particles impact on the oropharyngeal surfaces and get cleared. If cohesive forces are strong, shear does not separate carrier from drug and thus results in low deposition efficiency[15].

\section{Metered Dose Inhalers}

The mechanics of MDI involve a transient, cavitation turbulent fl uid that $\mathrm{fl}$ ashes into rapidly evaporating droplets. MDIs remain a standard aerosolized delivery system which can produce an aerodynamic particle size of less than 5 microns with HFA propellants. HFApropelled MDIs are easily portable, tamper-proof, and multidose. They protect the remaining product from oxidation, light, and moisture, while providing a simple and economical technology with accurate liquid actuation by volume. The aerosol drug dose exits the MDI mouthpiece as a rapidly moving large droplet cloud. However, at about the distance of the back of the throat, the droplet diameter is reduced due to propellant evaporation, and a reasonable proportion of the "polydispersed" aerosol cloud is now small enough to penetrate the lung. A proportion of each "metered dose" is lost in the actuator mouthpiece, and a further proportion is lost in the oropharynx due to inertial impaction of the "ballistic portion" of the spray.

\section{Nanoparticle-Based Systems For Pulmonary Application \\ Solid Lipid Nanoparticles (SLN) and Solid Lipid Microparticles (SLM)}

Solid lipid nanoparticles (SLN) have extensively been studied for a long time for potential pulmonary drug delivery. SLN are nanoscale aqueous suspensions prepared from physiological lipids, primarily triglycerides and phospholipids. As the formulations are based on using physiological components, they are less toxic and, as a result, more acceptable for pulmonary drug delivery. Phospholipids are present ubiquitously in the deep areas of lung and are essential for the functioning of the breathing mechanism. Phospholipid-based surfactant proteins present at the alveolar surface are essential for maintaining optimal surface tension and reducing friction in the lung tissue[16].

For the treatment of lung infections, various drugs have been investigated. SLN with amikacin, an amino glycoside antibiotic, was manufactured using cholesterol as the lipid by a high pressure homogenization method[17].

\section{Polymeric Nano particles}

Polymers are gaining rapid importance for pulmonary drug delivery. Several polymers have been investigated for pulmonary application. Polymers have numerous advantages, like modified surface properties, high encapsulation of the drug and protection of the drug from degradation, prolonged drug delivery and a long shelf life. For therapeutic purposes, the most commonly used polymers include poly(lactic acid) (PLA), poly(lactic-co-glycolic acid) (PLGA), poly( $\varepsilon-$ caprolactone) (PCL), alginate, chitosan and gelatin 
Base[18]. These are modified in their chemical and surface properties in order to make them biodegradable[19]. Recently, from a study performed by Beck-Broichsitter and colleagues, the influence of polymeric nanoparticles on a pulmonary surfactant and its surface properties was assessed. The authors also compared the effect of synthetic and biodegradable polymeric nanoparticles on the pulmonary surfactant. They found dose-dependent changes in the surface tension of the pulmonary surfactant. Several studies have been performed using chitosan-based nanoparticles for protein and gene delivery.

Although polymeric particles may be biodegradable, their degradation rate must be analyzed with toxicity profiles in various in vitro, ex vivo and in vivo models.

\section{Liposomes}

Liposomes are an attractive drug delivery system, especially for pulmonary applications, as it is prepared primarily from phospholipids, which are inherent in lungs. They are prepared using lung surfactants, phospholipids, cholesterol, etc. Liposomes possess sustained release properties, which enable the maximum drug effect over a prolonged time period. In the 1990s, the first liposomal product was introduced, which was purified bovine surfactant (Alveofact $\AA$ ) for acute respiratory distress syndrome (ARDS) in infants by pulmonary instillation. Later, amphotericin Bloaded liposomes were introduced (Ambisome ${ }^{\circledR}$ ), yet not for pulmonary, but for parenteral application. Inhaled liposomes are still a challenge. Maintaining the critical physical properties of liposomes after nebulization holds the key for a successful liposomal product[20].

Polymer-Based Nano particulate Delivery System Polymeric nanoparticles are colloidal carriers ranging in size from 10-1000 $\mathrm{nm}$. The smaller size helps in targeting and maintaining the encapsulated particles. It represents an attractive means of delivering the proteins. The polymer material tends to transform the physicochemical characteristics such as the drug release properties, zeta potential, and hydrophobicity. In the case of vaccines, the formulation of the particulate antigen delivery system is the choice of the polymer which must be safe, biocompatible, and biodegradable for use. The biodegradability is necessary for the release of the antigen and to avoid the surgical step for the recovery of the depleted system. Various types of the polymers used for the formulation of the nanoparticles are chitosan, alginates, PLGA, and PLA. The natural polymers like chitosan, gelatin, albumin and sodium alginate avoid the toxicological problems associated with the use of the synthetic polymers[21].

\section{Polysaccharide-Based Nano particulate Delivery System}

Chitosan is the mucoadhesive cationic polysaccharide that is the copolymer of the alpha glucosamine and Nacetyl-d-glucosamine. It is the most abundant biopolymer after cellulose, is made up of deacetylation of chitin, and is cheap, non-toxic, biodegradable, and biocompatible. It is generally used for the mucosal delivery of bio molecules due to its property of bioadhesiveness and high protein-binding efficiency. The use of chitosan for the administration of proteins to the lungs by the aerosol system has been observed and evaluated in many of the studies. The densities of noncross linked and glutraldehyde-crosslinked chitosan microspheres closely matched to the HFA propellant and found non crosslinked and glutraldehyde chitosan to be potential carriers of the protein molecules to the lungs .The production of chitosan nanoparticles has been demonstrated having a size range from $50-300 \mathrm{~nm}$ with small size dispersion. The nanoparticle size is dependent on the concentration of the dissolved polymer and on the size of the aerosol droplets. Chitosan was included in the formulation as a potential protective agent for the delivery of lactase dehydrogenase to the lungs using a nebulizer and thus chitosan was considered to be an essential excipient in the preparation of the stable formulations of the proteins for jet nebulization[22,23].

Alginates are mucoadhesive non-immunogenic natural polymers. They consist of 1,4 linked-D-mannuronic acid, $\alpha$ - L-guluronic acid, and the alternating blocks.. They gel in the presence of counter ions such as $\mathrm{Ca}$, which is applied for cell immobilization, delivering drugs and antigens due to non-toxicity and biodegradability. Aliginate nanoparticles have been prepared for combined delivery of antitubercular drugs such as isoniazid (INH), rifampicin (RIF), pyrazinamide (PZA). Developed systems have demonstrated high drug encapsulation effi ciency within the respirable range[24].

Hyaluronic acid (HA) is a polyanionic non sulfated polysaccharide that consists of N-acetyl-Dglucosamine and beta-glucoronic acid. HA is distributed widely in vertebrates and presents as a component of the cell coat of many strains of bacteria. One of the chief components of the extracellular matrix, hyaluronan, contributes significantly to cell proliferation and migration, and may also be involved in the progression of some malignant tumors. Due to its high biocompatibility, muco adhesitivity, and its common presence in the extracellular matrix of tissues, hyaluronan is gaining popularity in drug delivery systems[25]. 
Carboxy Methyl Cellulose (CMC) is anionic, biodegradable, and linear polymer cellulose ether. Carboxymethyl cellulose is a cellulose derivative with carboxymethyl groups $(-\mathrm{CH} 2-\mathrm{COOH})$ bound to some of the hydroxyl groups of the glucopyranose monomers that make up the cellulose backbone. It is one of the most versatile of water-soluble hydrocolloids and has a number of important properties including solubility, rheology, adsorption on surfaces, etc. CMC is pseudoplastic by nature and can show thixotropic and essentially non-thixotropic rheology. Besides controlling the rheology, CMC is also known to be an excellent excipient for spray-dried formulation. Similarly, many studies demonstrated that addition of CMC in the aerosol particulate formulations can increase the permeation, transfection, and internalization of therapeutics within the mucosal cells and retard the release of loaded drugs/biomolecules. Sodium carboxymethylcellulose has been used as a spray-drying excipient in the preparation of inhalable formulations of proteins[26].

Cyclodextrins are cyclic polymers of alpha-Dglucopyranose made up of sugar molecules bound together in a ring (cyclic oligosaccharides). Cyclodextrins are able to form host-guest complexes with hydrophobic molecules given the unique nature imparted by their structure. Cyclodextrins have an ability to alter physical, chemical, and biological properties of guest molecules through the formation of inclusion complexes. Cyclodextrin complexes can be used to enhance drug delivery through biological barriers without affecting their barrier function, a property which makes cyclodextrins ideal penetration enhancers for intranasal drug delivery. Cyclodextrins can also act as solubilizers for lipophilic waterinsoluble drugs, making it possible to formulate such drugs in aerosol formulations. Furthermore, cyclodextrin complexation can stabilize drugs that are chemically unstable in aqueous solutions, and decrease drug irritation after pulmonary application[27].

\section{ETHICS}

Polymeric NPs have been developed as effective carriers for delivery of various therapeutics and/or diagnostics for management of pulmonary infections due to their abilities to overcome drug resistance and to improve pharmacokinetics and bio distribution profiles of the administered drugs, thereby maximizing direct delivery and retention at the sites of infection while minimizing systemic exposure. Smart features also enable NPs to selectively release the loaded cargoes in specific microenvironments, disease sites, or other target sites in the body. However, there remain several challenges in the development of clinical nano medicines for treatment of lung infections, including those associated with the conditions experienced during circulation and/or upon reaching the lung environment[28].

\section{Physicochemical Characterization for Nano} particle-Based Systems

Particle Size and Zeta Potential Measurements Particle size and zeta potential measurements are important for the characterization of nanoparticles in order to ensure the optimal particle size distribution and polydispersity index (PDI). The most commonly used particle size measurement techniques include photon correlation spectroscopy (PCS) and laser diffraction (LD). PCS normally measures particles ranging between a few nanometers to a maximum of 3 $\mu \mathrm{m}$. The PCS technique measures the scattering of light by the dispersed particles, which move due to Brownian motion. PCS can also measure the PDI, which determines the uniformity of the particles. A higher value of PDI (above 0.2) normally indicates multiple sizes of particles in the given formulation. Hence, the smaller the PDI, the more uniformly the particles are distributed within the formulation. The LD technique measures particles with a bigger size and is based on the measurement of the diffraction angle depending on the particle radius. LD measures particles from the nanometer to a few millimeter size ranges[29] $]^{29}$.

Differential Scanning Calorimetry (DSC) Differential scanning calorimetry (DSC) is one of the most important methods for the analysis of polymorphic changes in a lipid matrix. Structural changes in the lipid matrix provide information regarding the stability of the formulation over time. Melting and recrystallization curves are appropriate parameters to determine polymorphic changes in lipid matrices[30].

\section{$\mathrm{X}$ - ray Diffraction}

Along with DSC, X-ray diffraction is essential for analyzing the crystal structure and spacing in the lipid lattice. The incorporation of an API influences the lipid/polymer structure and spacing of the lattice. This method provides information of the patterns in the spacing and changes in the lipid/polymer structure and crystallinity can be mapped along with DSC. Hence, it is recommended to use both techniques simultaneously when analyzing any lipid-based formulations[31]

\section{Microscopical Techniques/Particle Morphology}

The morphology of the nanoparticles can be examined by using transmission electron microscopy utilizing different techniques suitable for specific particles. Freeze-fracture, negative staining and cryogenictransmission methods can be adapted according to the type of particles. The interpretation of the morphology of the particle gives an idea about the structure, shape and alignment of the particles in the formulation[32] 


\section{Cell- and Animal-Based Studies}

Several studies have been performed for the toxicity analysis of the nanoparticle systems in different cell lines, tissue models, as well as animal models. Toxicity testing is essential in order to determine the lethal dose, as well as the therapeutic window of the drug-loaded nanoparticles. Apart from toxicity testing, therapeutic efficacy is also essential, and several models have been established that illustrate various disease conditions. Many in vitro models have been established using epithelial cells from the respiratory tract for testing nanoparticles after inhalation. Models involving an air liquid interface (ALI) have been extensively used to study the effects of various formulations intended for pulmonary application[33]

In vitro cell culture models are essential for the primary testing of any formulation before proceeding towards ex vivo and in vivo testing. In vitro cell models offer numerous advantages, such as continuous cell lines, easy handling and availability in large numbers, which offers the user many possibilities for designing multiple experiments simultaneously and, hence, limits the use of live animals. Different cell models derived from pulmonary epithelium from murine and human tissues have been established over the last two decades. Ex vivo lung tissue models have been used extensively along with the in vitro and in vivo models. Ex vivo models can be advantageous for studying drug transport mechanisms across lung tissue and can provide information with respect to in vitro-in vivo correlation. Different ex vivo models were established, such as isolated perfused lung (IPL) and precision cut lung slices (PCLS). IPL are prepared from mostly murine models, i.e., rats. Mice are seldom used, owing to their small size and the difficulty in the isolation procedure. Other animals commonly used include rabbits and guinea pigs. The IPL model includes a complete lung that has been isolated from the body and immersed in an artificial system that resembles physiological conditions[34]

In vivo models involve whole animals for drug absorption and disposition studies in the lungs. The commonly used animals in in vivo experiments include small rodents, like mice, rats and guinea pigs. Mice being much smaller than the others are challenging for the removal of blood samples and lung dosimetry. Other larger and much more expensive animals include pigs, rabbits, sheep and monkeys, where studies with regard to inhalation pharmacokinetics, formulation and device efficiency are concerned. The most commonly used method is intratracheal instillation. In intratracheal instillation, the animal's trachea is exposed, and through an endotracheal tube, an incision is made between the tracheal rings. Through this bifurcation, a typical volume between $10-200 \mu \mathrm{L}$ of an aqueous suspension or solution of the test formulation is instilled using a micro syring . Apart from intratracheal instillation, animal can be directly exposed to the aerosol in a chamber where the nose of the animal is fixed, and then, the animal inhales the released aerosol (the nose only model). After exposure for a specified time, the animal is sacrificed, and the broncho alveolar lavage fluid (BAL) is analyzed for the desired components[35].

In vitro and ex vivo toxicity testing involves many assays for assessing the toxicity of drug formulation. Different cell targets can be utilized, such as mitochondria, cell membrane integrity, nuclei staining, lysosomal activity and DNA ladder assays for apoptosis cell death mechanisms, etc. The 4,5dimethylthiazol-2-yl)-2,5-diphenyltetrazolium bromide (MTT) assay has been established for more than two decades as the standard for cell viability measurements in a variety of cell lines. Based on the similar principle of the metabolic activity of mitochondria, other assays involving tetrazolium salts, resazurin and the neutral red test can also be used to assess cell viability[36] Recent Advances in The Pulsatile Drug Delivery Nanotechnology has potential in the development of novel and effective delivery of drugs within lungs. Different strategies have been utilized for pulmonary delivery of drugs, including the use of lipid-based delivery systems (liposomes, ISCOMs, SLNs), use of polymeric matrix (PLGA, poly caprolactone, cynoacrylates, gelatin), development of polysaccharide particulates (chitosan, alginates, Carbopol, etc.), biocompatible metallic inorganic particles (iron, gold, zinc), etc. This paper reviews various nanopaticulate approaches in the form of lipids, polymers, metals, polysaccharides, or emulsions based for pulmonary drug delivery that could provide an increased biological efficacy and better local and systemic action. Polymeric NPs have been developed as effective carriers for delivery of various therapeutics and/or diagnostics for management of pulmonary infections due to their abilities to overcome drug resistance and to improve pharmacokinetics and biodistribution profiles of the administered drugs, thereby maximizing direct delivery and retention at the sites of infection while minimizing systemic exposure. Smart features also enable NPs to selectively release the loaded cargoes in specific microenvironments, disease sites, or other target sites in the body. However, there remain several challenges in the development of clinical nano medicines for treatment of lung infections, including those associated with the conditions experienced during circulation and/or upon reaching the lung environment.

Tahilani \& Chatterjee ASIAN PACIFIC JOURNAL OF NURSING AND HEALTH SCIENCES, 2021; 4(1):22-34 


\section{Metal-Based Nanoparticle Delivery}

Iron particles are of considerable interest for application in nanotechnology-related fi elds. Iron nano particles are sub micrometer particles of iron metal. They are widely used in medical, diagnostic, and laboratory applications. They are highly reactive because of their large surface area. In the presence of oxygen and water, they rapidly oxidize to form free iron ions. Iron is a highly redox-active transition metal, thus the safety of iron nano materials needs to be studied. Super paramagnetic iron oxide nano particles showed potential in magnetic targeting of inhaled aerosols within the lung. These particles are also used as contrast agents in magnetic resonance imaging (MRI). The nebulized droplets in the inhalation chamber had mass median aerodynamic diameter (MMAD). MRI studies have shown the in vivo distribution super paramagnetic iron oxide nano particles into lungs[37].

Colloidal gold is a suspension (or colloid) of sub micrometer-sized particles of gold in a fl uid, usually water. Gold nano particles have unique optical, electronic, and molecular-recognition properties, and are widely used in the areas of science, engineering, or medicine. Gold NPs have properties such as chemical stability, high electron density, and affinity to biomolecules, making them drug carriers and a tool for diagnosis. Different physicochemical properties of nano-sized gold particles have raised substantial concerns about the safety of gold nanoparticles in the body. During the past decades, various aspects of the interaction between gold nanoparticles and pulmonary/lungs structures have been investigated. Preliminary cytotoxic studies have shown that suspensions of well-dispersed $50 \mathrm{~nm}$ and $250 \mathrm{~nm}$ particles, as well as their agglomerates, produced very mild pulmonary inflammation at the same mass-based dose. Studies revealed that single $50 \mathrm{~nm}$ gold particles do not pose a greater acute hazard than their agglomerates or slightly larger gold particles when using pulmonary inflammation as a marker for toxicity[38]

Studies have been done in order to develop in vitro screening assays to determine lung hazard potential of nanomaterials involving three major objectives: a) to compare lung toxicity impacts of nanoscale $(\mathrm{NZnO})$ vs fi ne zinc oxide (FZnO) particulates; b) assess predictability of in vitro cell culture systems; and c) compare effects of instillation vs inhalation exposures in rats. Rats were exposed in vivo either by intratracheal instillation to 1 or $5 \mathrm{mg} / \mathrm{kg}$ of nanoscale or fi ne size zinc oxide particle types or by inhalation to aerosols of 25 or $50 \mathrm{mg} / \mathrm{m} 3$ for 1 or $3 \mathrm{~h}$. Lung infl ammation, cytotoxicity, and histopathological endpoints were assessed at several time points

postexposure[39].

\section{Nanotoxicology}

Nanomaterial research is focused on the medical applications of nanotechnology, whereas side-effects with nanotechnology use are not taken into consideration during the engineering process. The available methods, such as physiologically based pharmacokinetic modeling or predictive structureactivity relationships, assess the toxicity and risk associated with specific nano materials. This can be achieved through an innovative combination of toxicology, risk assessment modeling, and tools developed in the fi eld of mult icriteria decision analysis (MCDA) Recent applications of nano science include the use of nano-scale materials in electronics, catalysis, and biomedical research. Among these applications, biological processes such as blood coagulation control and multimodal bio imaging are also included, which has brought about a new and exciting research field called nano biotechnology. Biotechnology involves the manipulation of macroscopic biological systems such as cells and mice in order to understand why and how molecular-level mechanisms affect specifi c biological functions, e.g., the role of APP (amyloid precursor protein) in Alzheimer's disease (AD) [115]. Nanoparticles have been developed for many applications, including nanomedicines, cosmetics, food additives, and water purifi cation. Because of thehe potential for exposure of humans and the environment to nanoparticles, it is important to assess their safety, especially if nanotechnology is to reach its full potential. $\mathrm{N}$ anoparticles vary in terms of physicochemical characteristics such as size, surface area, charge, shape, surface chemistry, and contaminants. The current focus of nanotoxicology appears to be assessment of factors that drive toxicity, therefore providing information for risk assessment, hazard prevention, and future product design. Size effects particle uptake by cells and penetration across cell barriers, while surface area is related to the potential of particles to generate lung infl ammation. Shape, especially fi ber shape, appears to promote infl ammation, fi brosis, and granuloma formation in vivo. Positively charged particles are more toxic than negative particles, and some particles appear to leach soluble toxic components[40].

There are many organizations and much research supporting the development of nanotechnology. Some of them have also participated in proposing regulations to improve the protection of human health and the environment. These organizations or research centers are mainly supported by government sources and play

Tahilani \& Chatterjee $\quad$ ASIAN PACIFIC JOURNAL OF NURSING AND HEALTH SCIENCES, 2021; 4(1):22-34 
an essential role in performing or supporting nanotechnology research, including the basic research on nanotechnology, the applications of nanotechnology, safety assessment of nano materials, and the development of regulatory control [41].

\section{Current and Future Prospects}

The marked biotechnological advances observed in recent decades resulted in the appearance of many protein-based drugs. Fundamentally, the oral delivery of these molecules is prevented by the degrading effect of abundant protease content, and the possibilities of delivery are essentially reduced to injection-based strategies. However, this approach is more expensive and not appreciated by the patients, mainly due to the discomfort associated with the administration, but also because of eciated by the patients.

The pulmonary route thus appears as a sound alternative when a systemic effect is desired,but protein-based drugs also find applications in the treatment of local lung diseases.

Solid lipid nanoparticles (SLN) were also proposed for this, and were reported to provide homogeneous distribution through the lung upon delivery to diabetic rats by nebulization, showing a relative bioavailability of insulin of $22.3 \%$ comparing with subcutaneous injection. An approach similar to that referred above of chitosan nanoparticles microencapsulated in mannitol microparticles was later reported for the systemic delivery of calcitonin. The inhalable carriers had mass median aerodynamic diameter (MMAD) of $2.7 \mu \mathrm{m}$ and fine particle fraction (FPF) of 64\%, the latter representing the fraction of particles with an aerodynamic diameter lower than $5 \mu \mathrm{m}[42]$.

\section{Delivery of Antibiotics}

The delivery of antibiotics to the lung seems a very reasonable approach in the treatment of infections that are based in that organ. In fact, the most common routes of delivery of antibiotics are the oral and parenteral, even if the treatment of respiratory infections is intended. Addressing local lung infections requires reaching effective concentrations of the drug in the organ, which implies the administration of significantly high doses and a general exposure of the organism to the drugs. The direct administration to the infection site would, thus, permit using lower doses and avoid or decrease systemic exposure, with the consequent reduction in systemic side-effects.

Antibiotic resistance has been, for many years, one of the greatest public health problems. The increasing misuse of these molecules, ever since their discovery, has been making bacteria progressively resistant, by means of the development of specific cellular mechanisms. This has been continuously and consistently posing are new challenge to the treatment of infectious diseases[43].

\section{Applications in Cancer Therapy}

The WHO refers to lung cancer as one of the most lethal cancers . In 2018, $18.4 \%$ of cancer-related deaths were a result of lung cancer, and the number of new cases $(11.8 \%)$ was one of the highest, on par with breast cancer. WHO has a set of goals to fight cancer aggressively, and the development of new strategies in cancer treatment is a worldwide priority. Lung cancer can be categorized into non-small cell lung cancer (NSCLC) and small cell lung cancer (SCLC).

The scientific community has been working to develop more targeted therapies, which is facilitated by the increasing information on molecular pathways, specific receptors and the cancer microenvironment, enabling different treatment approaches. Although the intravenous route is the most frequently used to deliver anticancer drugs, including in lung cancer, the use of the lung route is an alternative yet to be fully explored in lung cancer therapy. This approach would allow a more targeted delivery, directly reaching the affected area, possibly with higher effectiveness than that provided by systemic delivery.

The number of nanocarriers proposed for an application in lung cancer mediated by lung delivery is high. In most cases, a therapeutic effect is envisaged, but some of the works address diagnostic purposes[44] Pharmaceutical technology has been playing a vital role on medicine, as it allows us to explore different materials and their combinations to prepare drug carriers, and to further endow these with better properties that enable us to reach the desired target sites, ending up with therapeutic success. Many questions arise around the topic of nanocarrier-based lung drug delivery. Scientists have given many and varied answers in attempt to address all the rising issues, finding alternatives and engineering adequate systems to fulfill requirements and needs. One of the concerns is always the fate of the drug. In lung drug delivery, the objective is sometimes to retain the drug in the lung as happens in local delivery approaches, thus minimizing the systemic absorption. In other cases, a systemic effect is the desired outcome and the carriers are engineered to avoid retention. The options to address therapeutic demands are varied, as seen by the plethora of systems, alternatives and engineering possibilities described through the review. As expected, the global analysis reveals that most of the works focus on the use of the lung route to attain local rather than systemic effects. It was also verified that, from the three main topics explored in the review, the

Tahilani \& Chatterjee ASIAN PACIFIC JOURNAL OF NURSING AND HEALTH SCIENCES, 2021; 4(1):22-34 
delivery of anticancer drugs is among the most prominent focuses in the literature, which is justified by the severity of the numbers associated with this disease, the increasing number of patients, and the lack of therapeutic options, besides the marketing appeal of cancer therapies[45].

\section{Conclusion}

Pulmonary drug delivery is rapidly gaining importance, due to the multiple advantages. Lungs offer a vast variety of advantages over conventional oral drug delivery. The large surface area and the elimination of the first past effect makes the absorption of drugs in the lungs faster. The size of these colloidal systems being in the nanometer range certainly gives them an advantage over conventional dosage forms. Poorly soluble drugs can be incorporated in various colloidal systems. Lipid-based colloidal systems namely, SLN and liposomes, have an added advantage owing to their physiological components in the formulation.

Nano particulate systems show great potential to deliver biologically active substances. The core of these systems encloses a variety of drugs, enzymes, and genes. Lipid, protein, polysaccharide-based nano particles are efficient modes for pulmonary delivery. Sophisticated electromechanical techniques overcome some common difficulties and ensure reproducibility of dose and delivery to the lungs. The new inhalation systems are certainly better than classic nebulizers or MDIs. Drug delivery to the lungs by aerosol inhalation can be accomplished in high dose by repetitive inhalation. Novel technologies provide significant clinical advantages in order to increase delivery efficiency and targeting of specific regions.

Nano medicine represents an extraordinary opportunity for the improvement of current therapies and for the development of innovative treatment options for $\mathrm{CF}$ previously considered hard or impossible to treat. The huge amount of research on NPs development as nanocarriers for gene and drug delivery directly to the lungs by inhalation has the opportunity to strongly modify symptomatic treatments for CF patients, as well as those based on CFTR modulators and gene therapies. Due to the peculiar environment in which the therapies have to operate, characterized by several biological barriers (pulmonary tract, mucus, epithelia, bacterial biofilm), the use of nanotechnologies to improve and enhance drug delivery or gene therapies seems to be an extremely promising way to be pursued. However, the road to a definitive realization is still long, although several nano systems were already tested with successful results with FDA approved drugs.

The area still needs to evolve in several topics before inhalable nanocarriers enter clinical trials. Not only the question of performing more realistic in vivo assays is determinant, but also the toxicological assessment plays a defining role. Helpful technologies have been arising, such as 3D printing, which was used on the printing of artificial airways that enabled the study of particle flowability and dose assessment. Lim et al. described this application in the neonate, showing a powerful tool to improve the ethics associated with formulation testing and to provide solutions for children born with respiratory complications

All in all, this review highlighted an integrative process that considers progress made at the level of basic science, which clarifies patho physiological aspects of each clinical condition, and the development of tools and strategies to reach the pharmacological targets. Many works were described with inhalable nano carriers that have displayed potential, even with the existing limitations. All the issues, however, point to a common objective of providing the knowledge to enable the engineering of nano carriers that will promote improved lung therapeutics.

\section{References}

1. C.Coester, P. Nayyar, and J.Samuel,"In vitro uptake of gelatin nano particles by murine dendritic cells and their intracellular localization European Journal of Pharmaceutics and Biopharmaceutics , 2006; 62(3): 306-314.

2. R. K. Verma and S. Garg, "Current status of drug delivery technologies and future directions,"Pharmaceutical Technology, vol.25,pp.1-14,2001.

3. Nanoparticles for drug delivery to the lungs. TRENDS in Biotechnology ,25:563-570. Finlay, W.H. (2005).

4. Gibaldi , s Drug Delivery Systems in Pharmaceutical Care. Bethesda, MD: American Society of Health System.

5. Labiris, N.R. and Dolovich, M.B. Pulmonary drug 5. delivery. Part I: Physiological factors affecting therapeutic effectiveness of aerosolized medications. Br J Clin Pharmacol ., 2003;56 : 588-599.

6. Tena, A.F.; Clarà, P.C. Deposition of inhaled particles in the lungs. Arch Bronconeumol. 2012; 48(17): 240-246.

7. Yang, W.; Peters, J.I.; Williams, R.O. Inhaled nanoparticles-A current review. Int. J. Pharm. 2008;356(18): 239-247.

8. Patton, J.S. Mechanisms of macromolecule absorption by the lungs. Adv. Drug Deliv. Rev. 1996; 19: 3-36.

9. Patton, J.S.; Brain, J.D.; Davies, L.A.; Fiegel, J.; Gumbleton, M.; Kim, K.-J.; Sakagami, M.; Vanbever, R.; Ehrhardt, C. The particle has 
landed-Characterizing the fate of inhaled pharmaceuticals. J. Aerosol Med. Pulm. Drug Deliv. 2010;23:S71-S87.

10. Anderson, M.W., Orton, T.C., Pickett, R.D. and Eling, T.E. 7. Accumulation of amines in the isolated perfused rabbit lung. J Pharmacol Exp. Ther 1974;189 : 456-466.

11. 11 D ollery, C.T. and Junod, A.F. Concentration of proponol in isolated, perfused rabbit lung. J. Pharmacol ,1976;57:67-71.

12. 12 J orfeldt, L., Lewis, D.H., Lofstrom, J.B. and Post, C. Lung uptake of lidocaine in healthy volunteers. Acta Anaesth Scand , 1979;23 : 567574.

13. R oerig, D.L., Kotrly, K.J., Dawson, C.A., Ahlf, S.B., 10. Gualtieri, J.F. and Kampine, J.P. First pass uptake of verapamil, diazepam and thiopental in the human lung. Anaesth Analg, $1989 ; 69: 461-466$.

14. Suhara, T., Sudo, Y., Yoshida, K., Okubo, Y., Fukuda, 11. H., Obata, T., Yoshikawa, K., Suzuki, K. and Sasaki, Y. The limiting role of mucus in drug absorption: Drug permeation through mucus solution. Int J Pharm 1998; 126:1.

15. Finlay, W.H. (2005). 2. The Mechanics of Inhaled Pharmaceutical Aerosols: An Introduction . London: Academic Press.

16. Beck-Broichsitter, M.; Ruppert, C.; Schmehl, T.; Guenther, A.; Betz, T.; Bakowsky, U.; Seeger, W.; Kissel, T.; Gessler, T. Biophysical investigation of pulmonary surfactant surface properties upon contact with polymeric nanoparticles in vitro. Nanomed. Nanotechnol. Biol. Med. 2011;7:341-350.

17. Varshosaz, J.; Ghaffari, S.; Mirshojaei, S.F.; Jafarian, A.; Atyabi, F.; Kobarfard, F.; Azarmi, S. Biodistribution of amikacin solid lipid nanoparticles after pulmonary delivery. BioMed. Res. Int. 2013;2013: 1-8.

18. Menon, J.U.; Ravikumar, P.; Pise, A.; Gyawali, D.; Hsia, C.C.W.; Nguyen, K.T. Polymeric nanoparticles for pulmonary protein and DNA delivery. Acta Biomater. 2014, in press, doi:10.1016/j.actbio.2014.01.033

19. Beck-Broichsitter, M.; Ruppert, C.; Schmehl, T.; Günther, A.; Seeger, W. Biophysical inhibition of synthetic vs. naturally-derived pulmonary surfactant preparations by polymeric nanoparticles. Biochim. Biophys. Acta 2013; 1838:474-481.

20. Adler-Moore, J.; Proffitt, R.T. AmBisome: Liposomal formulation, structure, mechanism of action and pre-clinical experience. J. Antimicrob. Chemother. 2002; 49: 21-30.

21. 21 itt, C.G. (1990). Poly- $\varepsilon$ caprolactone and its copolymers. In: Langer, R. (ed.), Biodegradable Polymers as Drug Delivery Systems . New York: Marcel Dekker, p. 71.

22. Shoyele, S.A. and Slowey, A. Prospects of formulating proteins/peptides as aerosols for pulmonary drug delivery. Int J.of Pharmaceutics 2006;314 : 1-8.

23. lbasarah, Y.Y., Somavarapu, S. and Taylor, K.M.Stabilizing protein formulations during airjet nebulization. Int J Pharm , 2010;402 : 1405.

24. Ahmad, Z. and Khuller, G.K. Alginate- based 70. sustained release drug delivery systems for tuberculosis. Expert Opin Drug Deliv, 2008; 5 : 1323-34.

25. Frasher, J.R.E., Laurent, T.C., Laurent, U.B.G. Hyaluronan: Its nature, distribution, functions and turnover. Journal of Internal Medicine, 1997; $242: 27-33$.

26. Kalalipour, M., Najafabadi, A.R., Gilani, K., Esmaily, 87. H. and Tajerzadeh, H. Effect of dimethylbeta-cyclodextrin concentrations on the pulmonary delivery of recombinant human growth hormone dry powderin rats. J Pharm Sci, 2008;97 : 5176-85.

27. Bonacucina, G., Martelli, S. and Palmieri, G.F. Rheological, mucoadhesive and release properties of Carbopol gels in hydrophilic cosolvents. Int. J. Pharm , 2004;282: 115- 130.

28. Roberts RA, Shen T, Allen IC, Hasan W, DeSimone JM, Ting JPY. Analysis of the murine immune response to pulmonary delivery of precisely fabricated nano- and microscale particles. PLoS ONE. 2013; 8:e62115.doi: 10.1371/journal.pone.0062115

29. 29 Mueller, R. Solid lipid nanoparticles (SLN) for controlled drug delivery - A review of the state of the art. Eur. J. Pharm. Biopharm. 2000;50:161-177.

30. Bunjes, H. Lipid nanoparticles for the delivery of poorly water-soluble drugs. J. Pharm. Pharmacol. 2010;62:1637-1645.

31. Bunjes, H. Structural properties of solid lipid based colloidal drug delivery systems. Curr. Opin. Colloid Interface Sci. 2011; 16: 405-411.

32. Bunjes, H.; Unhruh, T. Characterization of lipid nanoparticles by differential scanning calorimetry, X-ray and neutron scattering. Adv. Drug Deliv. Rev. 2007;59:379-402. 
33. Scherließ, R. The MTT assay as tool to evaluate and compare excipient toxicity in vitro on respiratory epithelial cells. Int. J. Pharm. 2011; 411: 98-105.

34. Sakagami, M. In vivo, in vitro and ex vivo models to assess pulmonary absorption and disposition of inhaled therapeutics for systemic delivery. Adv. Drug Deliv. Rev. 2006; 58:10301060.

35. Foster, K.A.; Avery, M.L.; Yazdanian, M.; Audus, K.L. Characterization of the Calu-3 cell line as a tool to screen pulmonary drug delivery. Int. J. Pharm. 2000;208:1-11.

36. Arora, S.; Rajwade, J.M.; Paknikar, K.M. Nanotoxicology and in vitro studies: The need of the hour. Toxicol. Appl. Pharm. 2012;258:151165.

37. Martin, A.R., Thompson, R.B. and Finlay, W.H. MRI 100. measurement of regional lung deposition in mice exposed nose-only to nebulized superparamagnetic iron oxide nanoparticles . J Aerosol Med Pulm Drug Deliv, 2008;21 : 335-42

38. Kessinger, C.W., Peyton, M., Minna, J.D., Brown, K.C. and Gao, J. MRI-visible micellar nanomedicine for targeted drug delivery to lung cancer cells. Mol Pharm, $2010 ; 7: 32-40$.

39. Hauluhn, J. and Rosenbruch, M. Inhalation toxicity 111. of propineb. Part I: Results of subacute inhalation exposure studies in rats. Inhal Toxicol , 2003;15:411-34.

40. Shvedova, A., Valerian, E.K. and Bengt, F. Close 116. encounters of the small kind:

Source of Support: Nil

Conflict of Interest: Nil
Adverse effects of man made material interfacing with the nano-cosmos of biological systems. Annu Rev Pharmacol Toxicol , 2010;50 :63-88.

41. Tsytsikova, L. (2009). 118. Global List of Organizations and Efforts Related to Nanotechnology, Nanoscience, Nanomaterials, and Food and Agriculture Products. Available at: http://www.ilsi.org/NorthAmerica/Documents/F OOD $\%$ 20CHEMICAL $\% 20$ SAFETY/Global $\% 20$ List $\% 20$ of $\% 20$

Organizations $\% 20$ and $\% 20$ Efforts $\% 20$ Related $\% 2$ 0to\%20 Nanotechnology.pdf. Accessed on 18 March 2011.

42. Buttini, F.; Colombo, G.; Kwok, P.C.L.; Wui, W.T. Chapter 6-Aerodynamic assessment for inhalation products: Fundamentals and current pharmacopoeial methods. In Inhalation Drug Delivery: Techniques and Products; John Wiley \& Sons: Chichester, UK, 2013; pp. 91-119.

43. Brinkac, L.; Voorhies, A.; Gomez, A.; Nelson, K.E. The threat of antimicrobial resistance on the human microbiome. Microb. Ecol. 2017; 74:1001-1008.

44. Mejias, J.C.; Roy, K. In-vitro and in-vivo characterization of a multi-stage enzymeresponsive nanoparticle-in-microgel pulmonary drug delivery system. J. Control. Release 2019; 316:393-403.

45. Gaspar, R.; Duncan, R. Polymeric carriers: Preclinical safety and the regulatory implications for design and development of polymer therapeutics. Adv. Drug Deliv. Rev. 2009; 61:1220-1231. 